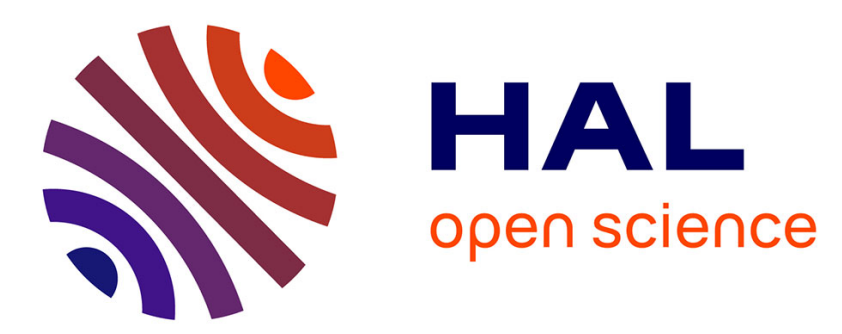

\title{
Algebraic observer for a class of switched systems with Zeno phenomenon
}

Gang Zheng, Lei Yu, Driss Boutat, Jean-Pierre Barbot

\section{To cite this version:}

Gang Zheng, Lei Yu, Driss Boutat, Jean-Pierre Barbot. Algebraic observer for a class of switched systems with Zeno phenomenon. 48th IEEE Conference on Decision and Control, CDC 2009, Dec 2009, shanghai, China. inria-00423493

\section{HAL Id: inria-00423493 https://hal.inria.fr/inria-00423493}

Submitted on 11 Oct 2009

HAL is a multi-disciplinary open access archive for the deposit and dissemination of scientific research documents, whether they are published or not. The documents may come from teaching and research institutions in France or abroad, or from public or private research centers.
L'archive ouverte pluridisciplinaire HAL, est destinée au dépôt et à la diffusion de documents scientifiques de niveau recherche, publiés ou non, émanant des établissements d'enseignement et de recherche français ou étrangers, des laboratoires publics ou privés. 


\title{
Algebraic observer for a class of switched systems with Zeno phenomenon
}

\author{
G. Zheng, L. Yu, D. Boutat and J-P. Barbot
}

\begin{abstract}
For a large class of switched systems with zeno phenomenon, classical observer cannot be applied directly since the terms leading to zeno phenomenon are not derivable. However in this paper, by assuming that these terms are integrable in the less restrictive way, we can define a new output, with which algebraic observer can then be adopted to estimate the states of the studied switched systems with zeno phenomenon. For simplicity, the main idea is explained via normal forms, while it can also be extended to generic switched systems.
\end{abstract}

\section{INTRODUCTION}

Switched systems are widely used for modeling complex systems, such as biological systems and engineering systems, since they can complicatedly describe the interactions of a family of continuous-time or discrete-time dynamical systems [21]. During the study of the switched system, most generally we exclude the case where zeno phenomenon occurs.

However, zeno phenomenon is well-known in physical domain, for example the bouncing ball case leading to many theoretical developments and simulations [2], [15], [18]. The bouncing ball is an impact system, which may be seen as a switched system with jump at the transient. In this paper we have not treated the case of switched systems with state jump, but the observation of such system which, in our point of view, is a very challenge problem. Moreover, in the widely used theory of variable structure for the purpose of control design or observer design, for example the first order sliding mode [27] and high order sliding mode [6], [19], there exists also zeno phenomenon. Let us remark that a first order sliding mode can generate a chattering zeno phenomenon and high order sliding mode will generally produce a genuinely zeno phenomena [2]. Obviously, in practice, it is impossible to design a control law which commutes at an infinite frequency, and zeno phenomena is only approximated by hight frequency switching for systems driven by sliding mode control, nevertheless all methods developed in this paper also work for hight frequency switching. Some other links between zeno phenomenon and sliding mode can be found

G. Zheng is with Project ALIEN, INRIA Lille-Nord Europe, 50, avenue Halley, 59650 Villeneuve d'Ascq, France gang. zheng@inria. fr

L.Yu is with Signal Processing Laboratory, Electronic and Information School, Wuhan University, China, and ECS ENSEA, 6 Avenue du Ponceau, 95014 Cergy-Pontoise, and Project ALIEN, INRIA, France. lei.yudensea. fr

D. Boutat is with ENSI de Bourges PRISME, 10 Boulevard de Lahitolle, 18020 Bourges, France driss . boutateensi-bourges. fr

J-P. Barbot is with ECS ENSEA, 6 Avenue du Ponceau, 95014 CergyPontoise, and Project ALIEN, INRIA, France barboteensea. fr with respect to the homogeneous concept, see for example [12] and [20].

Since zeno phenomenon cannot be avoided in some cases, hence this paper focuses on the study of observer design problem for a class of switched systems with zeno phenomenon. As the existence of zeno phenomenon makes the trajectory of studied system non derivable since it is high frequency switching, traditional observer design techniques, such as sliding mode observer [24], high-gain observer [13], algebraic observer [4], cannot be applied directly. Consequently this paper proposes a new approach to design an algebraic observer for a class of switched systems with zeno phenomenon, where basic idea is to re-define a new derivable output with which numerical differentiation algorithm ([8], [22]) can be used to calculate its successive derivative, and at the same time the built-in low-pass filters of this algorithm can remove the influence of the high frequency signals (terms containing/causing zeno phenomenon). Since normal form represents a class of equivalent systems with the same properties, for the sake of simplicity we will explain our main idea based on normal forms.

This paper is organized as follows. The notation, motivations and the problem statements are given in section 2 . In section 3, we present our main idea which is to define a new output based on which an observer can be designed to estimate the states for a class of switched systems with zeno phenomenon. After that, we highlight the fact that an algebraic observer can be used in such situation. This paper ends up with an illustrative example for the purpose of highlighting the feasibility of the proposed method.

\section{Problem Statement}

Let us consider the following class of switched systems with zeno phenomenon:

$$
\left\{\begin{array}{l}
\dot{x}=f(x)+g(x) q=f(x)+\sum_{i=1}^{n} g_{i}(x) q_{i} \\
y=h(x)
\end{array}\right.
$$

where $x \in U \subset R^{n}$ is the set of admissible state, $y \in R$ is the measured output and vector fields $f: U \rightarrow R^{n}, g_{i}: U \rightarrow$ $R^{n}, q_{i}: R^{+} \rightarrow Q=\{0,1\}$ and $h: U \rightarrow R$ are sufficiently smooth.

In our study, $q$ is not considered as an unknown perturbation but as a known input, which leads to zeno phenomenon. Hence we assume that $q$ preserves the observability of system (1) since $q$ is a known input. If (1) has a relative degree equal to its dimension, then the observer design problem becomes trivial and lots of nonlinear observers can be applied directly. 
Hence this paper focuses only on one of the non trivial cases where system (1) has a relative degree smaller than $n$. Moreover, in this case if $q$ is derivable, the regularly weakly locally observability of system (1) implies the following expression

$$
x=\Psi\left(y, \dot{y}, \cdots, y^{(n-1)}, q, \cdots, q^{(k)}\right)
$$

with $k \in\{1, \ldots, n-2\}$. From (2), many observers, including algebraic observer, can be employed in order to estimate the state $x$. However, for the case where zeno phenomenon exists in system (1), $q$ is considered as a high frequent switched signal which leads to zeno phenomenon. Consequently $q$ is not derivable. Hence it is no longer possible to apply most of existing observers for (1) with zeno phenomenon, since the successive derivatives of $q$ in (2) do not exist.

So, strongly motivated by this point, this paper deals with the problem: First to find a new equation similar to (2) which contains only derivable elements, and after that from this new equation to design an algebraic observer for a class of switched systems in the form (1) with zeno phenomenon in order to estimate its states.

\section{MAIN RESULT}

In this paper, we consider the Henstock-Kurzweil integral (HK integral) [14][5], also known as the gauge integral, the generalized Riemann integral. Let us recall both the definitions related to Riemann integral and the HK integral in order to clarify their differences.

Definition 1: Let us consider a real function $f$ defined on an interval $[a, b] \subset R$. For any tagged partition $P$ of $[a, b]$ such as $a=x_{0}<x_{1}<x_{2}<\cdots<x_{n}=b$, and $\tilde{x}_{i} \in$ $\left[x_{i-1}, x_{i}\right] \subset[a, b]$, consider $F(P):=\sum_{i=1}^{n} f\left(\tilde{x}_{i}\right)\left(x_{i}-x_{i-1}\right)$, for any given function $\delta:[a, b] \rightarrow \stackrel{i=1}{R_{+}^{*}}, P$ is said to be a $\delta$-fine tagged partition of $[a, b]$ if $h_{i}:=x_{i}-x_{i-1}<\delta\left(\tilde{x}_{i}\right)$ where $\delta$ is called a gauge for each $i$.

Definition 2: A number $I$ is called the Riemann integral (respectively the HK integral) of $f:[a, b] \rightarrow R$ if for each constant $\varepsilon>0$, there exists a constant $\delta$ (respectively a function $\delta:[a, b] \rightarrow R_{+}^{*}$ ), such that whenever $P$ is a $\delta$-fine tagged partition of $[a, b]$, we have $|I-F(P)|<\varepsilon$.

Roughly speaking, the integral of $f$ on $[a, b]$ is obtained by approximating the region under the curve defined on $[a, b]$ as a union of small rectangles. The Riemann integral requires that all those rectangles depend on a constant $\delta$ while the HK integral uses more sophisticated $\delta$ ( $\delta$ depends on $\tilde{x}_{i}$ ). In fact, when a function $f$ oscillates more quickly at some points of the subinterval $[a, b]$, one has to tighten the step $h_{i}$ at these points in order to approximate more precisely the associated surface. It is possible by choosing $h_{i}<\delta\left(\tilde{x}_{i}\right)$ such that $\delta\left(\tilde{x}_{i}\right)$ is a sufficiently small positive value that depends on the place from where the rectangle of height $f\left(\tilde{x}_{i}\right)$ is considered.

According to the characteristics of zeno phenomenon, such as the Chattering Zeno (i.e. after some time the dwell time is exactly equal to zero) or the Genuinely Zeno (i.e. the dwell time is never equal to zero)[2], we make the following assumption:
Assumption 1: $q$ is HK integrable ${ }^{1}$ or HKP integrable ${ }^{2}$. Moreover its averages $q_{f}$ is measured via a low-pass filter of sufficiently large bandwidth on time interval $[0, c]$ where $c \in R^{+}$.

Based on Assumption 1, our main idea is to construct a new output $Y$ based on the knowledge of the old output $y$, $q$, the successive derivatives of $y$ and successive integrals of $q$ since $y$ is derivable until $q$ appears in its derivative, and $y$, $q$ are integrable, i.e.

$$
Y=\varphi\left(y, \int^{(m)} \rho(y) q, \frac{d^{k}}{d t^{k}} y\right)
$$

where $k \in\{1,2, \ldots, n-1\}$ represents the $k$ th derivative of $y$ before that $q$ appears, $\int^{(m)} \rho(y) q$ with $m \in\{1,2, \ldots, n-1\}$ represents the $m$ th HK integral of the variable $\rho(y) q$ where $\rho(y)$ a smooth function of $y$.

For the sake of clarity, we denote by $\left(\int \rho(y) q d t\right)^{\prime}$ the exact derivative of $\mathrm{HK}$ integral which is equal to $\rho(y) q$ and $\left(\int \widehat{\rho(y) q} d t\right)$ the practical derivative of HK integral which is equal to $\rho(y) q_{f}$. As it is highlighted hereafter numerical differentiation introduced by Fliess and Sira-Ramirez [25][22] gives the second solution of practical differentiation.

With the new defined output $Y$, if we can express the state $x$ of (1) as follows

$$
x=\Psi\left(Y, \dot{Y}, \cdots, Y^{(n-1)}, \chi, \dot{\chi}, \cdots \chi^{(k)}\right)
$$

where $\chi$ is a set of new known $k$ th derivable variables defined as function of integrals of $y$ and $q$ since they are integrable. From (3) it is possible to design an observer to estimate the states of system (1).

It is well-known that normal form represents a class of equivalent dynamical systems possessing the same properties, such as controllability [16] and observability [3]. Hence for the sake of simplicity, we will explain our main idea for certain normal forms.

\section{A. Normal form of [17]}

Throughout this paper, $L_{f}^{i-1} h$ for $1 \leq i \leq n$ denotes the $(i-1)^{t h}$ Lie derivative of output $h$ in the direction of $f$, and set $\theta_{i}=d L_{f}^{i-1} h$ as its differential. For (1), assume that for all $x \in U$, the pair $(y, f)$ is regularly weakly locally observable, i.e., the codistribution $\operatorname{span}\left\{d h, d L_{f} h, \cdots, d L_{f}^{n-1} h\right\}$ is of rank $n$. Let us consider the vector field $\tau_{1}$ defined in [17] as follows

$$
\left\{\begin{array}{l}
\theta_{i}\left(\tau_{1}\right)=0, \text { for } 1 \leq i \leq n-1 \\
\theta_{n}\left(\tau_{1}\right)=1
\end{array}\right.
$$

and by induction we define

$$
\tau_{k}=(-1)^{k-1} a d_{f}^{k-1}\left(\tau_{1}\right), \text { for } 2 \leq k \leq n .
$$

Then if the following conditions are satisfied [17]:

$$
\left\{\begin{array}{l}
{\left[\tau_{i}, \tau_{j}\right]=0} \\
{\left[\tau_{k}, g_{i}\right]=0}
\end{array}\right.
$$

\footnotetext{
${ }^{1}$ see also Denjoy-Khinchine integrable.

${ }^{2}$ Henstock-Kurzweil-Pettis Integral is a generalization of HenstockKurzweil Integral where a Flippov solution may be taken into account [26]
} 
for $1 \leq i \leq n, 1 \leq j \leq n$ and $1 \leq k \leq n-1$, then (1) can be transformed into the following normal form ${ }^{3}$ :

$$
\begin{cases}\dot{z}_{1} & =z_{2}+\alpha_{1}(y)+\beta_{1}(y) q \\ \vdots & = \\ \dot{z}_{n-1} & =z_{n}+\alpha_{n-1}(y)+\beta_{n-1}(y) q \\ \dot{z}_{n} & =\alpha_{n}(y)+\beta_{n}(y) q \\ y & =z_{1}\end{cases}
$$

where $z=\left(z_{1}, \cdots, z_{n}\right)^{T}, \alpha=\left(\alpha_{1}, \cdots, \alpha_{n}\right)^{T}, q$ is $n \times 1$ matrix and $\beta_{i}$ is $1 \times n$ matrix for $1 \leq i \leq n$.

As explained before, because of the existence of zeno phenomenon in the studied switched system, it is no longer possible to apply directly most of existing observers, including algebraic observer proposed in [4], to systems in the form (6). However, we will show that, by re-defining a new output for the form (6), it is still possible to apply the algebraic observer to estimate its states. And this possibility is based on the Assumption 1.

Due to Assumption 1, we can define the following measurable variables:

$$
\begin{aligned}
\chi_{i}(t) & =\underbrace{\int_{t-T}^{t} \cdots \int_{s-T}^{s}}_{i \text { times }} \beta_{i}(y(\xi)) q_{f} \underbrace{d \xi \cdots d s}_{i \text { times }} \\
& :=\int^{(i)} \beta_{i}(y(\xi)) q_{f} d \xi
\end{aligned}
$$

where $T$ represents the time windows used by algebraic observer when calculating the derivative of a signal. It is obvious that the $j$ th derivative of $\chi_{i}$ satisfies:

$$
\chi_{i}^{(j)}= \begin{cases}\int^{(i-j)} \beta_{i}(y(\xi)) q_{f} d \xi, & \text { if } j<i \\ \beta_{i}(y) q_{f}, & \text { if } j=i\end{cases}
$$

where $q_{f}$ implies the average value of $q$, measured thought a low-pass filter. Let us remark that the superscript $j$ should be smaller than or equal to the subscript $i$, i.e. $j \leq i$, in (8) since the term $\beta_{i}(y) q_{f}$ is not derivable. It is clear that $\chi_{i}^{(j)}$ for $1 \leq j \leq i \leq n$ is a measurable term. Consequently we can define a new output for the form (6) as follows:

$$
Y=y-\sum_{i=1}^{n} \chi_{i}
$$

Proposition 1: If form (6) is algebraically observable for all $q$ sufficiently derivable with the old output $y$, then it is also algebraically observable under the new output $Y$ define in (9). Moreover its states can be expressed as follows:

$$
z=\Psi\left(Y, \dot{Y}, \cdots, Y^{(n-1)}, \chi_{i}, \dot{\chi}_{i}, \cdots \chi_{i}^{(i-1)}\right)
$$

where $\chi_{i}$ is define in (7) for $1 \leq i \leq n-1$.

Proof: For (6), let us consider the first exact derivative of the new output $Y$ defined in (9), then we have

$$
\begin{aligned}
(Y)^{\prime} & =y^{\prime}-\beta_{1}(y) q_{f}-\sum_{i=2}^{n} \dot{\chi}_{i} \\
& =z_{2}+\beta_{1}(y)\left(q-q_{f}\right)-\sum_{i=2}^{n} \dot{\chi}_{i}
\end{aligned}
$$

\footnotetext{
${ }^{3}$ The equivalent relation considered here is by diffeomorphism and output injection of the form $\alpha(y)+\beta(y) q$.
}

In this equation, since $q_{f}$ is the average of $q$, then the term $\left(q-q_{f}\right)$ can be seen as noise since it varies with a very high frequency (zeno phenomenon). Then a low-pass filter can be implemented in order to remove this noise ${ }^{4}$, which therefore yields the estimate of $z_{2}$. So in this case we deal with practical derivative, i.e. replace $(Y)^{\prime}$ by $\dot{Y}$, and we obtain

$$
z_{2}=\dot{Y}+\sum_{i=2}^{n} \dot{\chi}_{i}
$$

According to the above equation, the exact derivative of $z_{2}$ gives:

$$
\begin{aligned}
(\dot{Y})^{\prime} & =\left(z_{2}\right)^{\prime}-\beta_{2}(y) q_{f}-\sum_{i=3}^{n} \ddot{\chi}_{i} \\
& =z_{3}-\beta_{2}(y)\left(q-q_{f}\right)-\sum_{i=3}^{n} \ddot{\chi}_{i}
\end{aligned}
$$

Analogously, because of the low-pass filter or practical derivative we obtain the estimate of $z_{3}$ :

$$
z_{3}=\ddot{Y}+\sum_{i=3}^{n} \ddot{\chi}_{i}
$$

Iteratively, with the same procedure, for the estimate of $z_{j}$ we have:

$$
z_{j}=Y^{(j-1)}+\sum_{i=j}^{n} \chi_{i}^{(j-1)}
$$

for $1 \leq j \leq n$. Finally, we can express the states of (6) as a function without $q$ as follows:

$$
z=\Psi\left(Y, \dot{Y}, \cdots, Y^{(n-1)}, \chi_{i}, \dot{\chi}_{i}, \cdots \chi_{i}^{(i-1)}\right)
$$

which implies that for example an algebraic observer can be applied to observe the states of (6) even if it has zeno phenomenon.

Remark 1: Let us remark that in the form (6), the term $\beta_{i}(y) q$ is not derivable, but integrable, and it allows us to introduce a derivable term $\chi_{i}$ which is certain integrals of the term $\beta_{i}(y) q_{f}$. The introduction of the term $\chi_{i}$ is for the purpose of compensating the effect of the term $\beta_{i}(y)\left(q-q_{f}\right)$. Consequently, the compensated residue of these two terms can be regarded as a noisy signal with high frequency, which allows us to use low-pass filters to remove its effect.

\section{B. More generic normal form}

Let us consider the following more generic normal form:

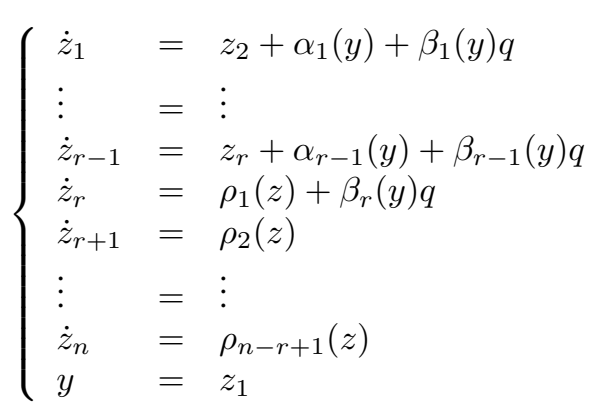

${ }^{4}$ The choice of filter bandwidth is easy where zeno phenomenon appears but may be difficult where the switching frequency is widely variable. 
where $z=\left(z_{1}, \cdots, z_{n}\right), q$ is $n \times 1$ matrix and $\beta_{i}$ is $1 \times n$ matrix for $1 \leq i \leq r$.. Compared to (6), (10) represents a larger class of switched system, including the case where (1) cannot be linearized with output injection into the form (6). The part that cannot be linearized with output injection is transformed to the part of zero dynamic in (10).

Remark 2: To seek the sufficient and necessary condition of transforming (1) into (6) is beyond the scope of this paper, however they can be deduced based on the same idea of [17]. It should be noted that we can only constructively determine $r \in[1, n$ [ vector fields from (4) and (5) whose Lie brackets are commutative, which implies the first $r$ states of (10) are linearizable with output injection. The undetermined vector fields corresponding to zero dynamics of (10) might be determined through commutative condition of Lie bracket between $g$ and these vector fields.

In order to design an observer for the form (10), we need firstly study its observability. As stated before, $q$ in the form (10) is regarded as a known signal, but we can only measure its average value because of its high fluctuation. The first part of (10) is linear with output injection, which is exactly in the form (6). As discussed in the last section, it is possible to define some measurable variables in the form (7) based on which a new output can be defined in the form (9) such that observers can be used to estimate $z_{i}$ for $1 \leq i \leq r$ of the normal form (10). In other words, it yields

$$
\bar{z}=\Psi\left(Y, \dot{Y}, \cdots, Y^{(r)}, \chi_{i}, \dot{\chi}_{i}, \cdots \chi_{i}^{(i-1)}\right)
$$

where $\bar{z}=\left(z_{1}, \cdots, z_{r}\right)^{T}, \chi_{i}$ and $Y$ can be defined as the form (7) and (9) for a certain $i \in\{1,2, \ldots, r\}$. Hence, let us consider the second part of the form (10), which can be written as follows:

$$
\left\{\begin{array}{l}
\dot{\tilde{z}}=\rho(\tilde{z}, \bar{z}) \\
\tilde{y}=\tilde{h}(\tilde{z}, \bar{z})=\rho_{1}(\tilde{z}, \bar{z})
\end{array}\right.
$$

with $\bar{z}=\left(z_{1}, \cdots, z_{r}\right)^{T}, \tilde{z}=\left(z_{r+1}, \cdots, z_{n}\right)^{T}, \rho(\tilde{z}, \bar{z})=$ $\left[\rho_{2}(\tilde{z}, \bar{z}), \cdots, \rho_{n-r+1}(\tilde{z}, \bar{z})\right]^{T}$ where $\bar{z}$ and $\tilde{y}$ are known with the help of the application of observer for the first part of (10).

Assumption 2: (12) is uniformally weakly locally observable with respect to $\bar{z}$.

Remark 3: Assumption 2 implies that there exists a smooth function $\tilde{\Psi}_{\bar{z}}$, such that the following equation satisfied:

$$
\tilde{z}=\tilde{\Psi}_{\bar{z}}\left(\tilde{y}, \dot{\tilde{y}}, \cdots, \tilde{y}^{(n-r)}\right)
$$

It is evident that (10) is locally weakly observable if Assumption 2 is fulfilled. Moreover, it is possible to design an observer to estimate the states of (10) according to (11) and (13).

Remark 4: Using the concept of normal form in order to explain our main idea is firstly for facilitating the explanation and secondly for establishing a uniform formula. The main idea presented in this section can, without any difficulty, be adapted to deal with other normal forms [28], [31], [29] with zeno phenomenon.

\section{Algebraic ObSERVER}

Since the time derivatives will amplify noise, hence for the proposed method, a low-pass filter should be firstly applied for the purpose of removing the noisy residue, and a numerical differentiation algorithm can then be employed in order to calculate its derivative. Thus, each signal should be equipped with a pair of filter and differentiator. In order to simplify the implementation, we use algebraic observer proposed in [4], which is based on the numerical differentiation technique proposed by Fliess et al in [25][22]. This algebraic approach has at least the following advantages:

- It provides explicit formulae, which can be directly implemented;

- It is non-asymptotic, which is a significant advantage for real-time applications;

- It does not require any assumption concerning the statistical distribution of the unstructured noise;

- ...

Mathematical foundation of this approach can be referred to [8], [9], [7]. Some applications of such method can be cited in [4], [30] for secure communication, [23] for adaptive input shaper design, [10] for switched system, [11] for model-free control.

Let $y(t)=x(t)+n(t)$ be a noisy observation on a finite time interval of a real-valued signal $x(t)$, the derivatives of which we want to estimate. Then replace the convergent Taylor expansion

$$
x(t)=\sum_{n \geq 0}^{\infty} a_{n} \frac{t^{n}}{n !}
$$

by its truncated Taylor expansion

$$
x_{N}(t)=\sum_{n \geq 0}^{N} a_{n} \frac{t^{n}}{n !}
$$

It reads in the operational domain as:

$$
s^{N+1} x_{N}(s)=s^{N} x_{N}(0)+s^{N-1} \dot{x}_{N}(0)+\cdots+x_{N}^{(N)}(0)
$$

And then annihilate the remaining coefficients $x_{N}^{j}(0), j \neq n$ by multiplying by linear differential operators of the form

$$
\Pi_{\kappa}^{N, n}=\frac{d^{n+\kappa}}{d s^{n+\kappa}} \cdot \frac{1}{s} \cdot \frac{d^{N-n}}{d s^{N-n}} \quad \kappa \geq 0
$$

It yields the following estimator for $x^{(n)}(0)$ :

$$
\frac{\tilde{x}_{N}^{(n)}(0)}{s^{\nu+n+\kappa+1}}=\frac{(-1)^{n+\kappa}}{(n+\kappa) !(N-n) !} \frac{1}{s^{\nu}} \Pi_{\kappa}^{N, n}\left(s^{N+1} \hat{x}\right)
$$

which is strictly proper whenever $\nu$ is of the form $\nu=N+$ $1+\mu$ with $\mu>0$. It should be noted that $\mu$ here represents the number of low-pass filter. After transferring both equations (15) into the time domain, and several calculations, the estimate of the $n^{t h}$-order derivative of $x(t)$ with the form 
as follows ${ }^{5}$.

$$
\begin{aligned}
\tilde{x}_{N}^{(n)}(0)= & A(\nu, n, \kappa, N) \sum_{i=0}^{N-n} B(i, n, N) \sum_{j=j_{0}}^{n+\kappa} C(i, j, n, \kappa) \\
& \int_{0}^{1}(1-\tau)^{\nu+\kappa-i-j-1}(-\tau)^{i+j} x(T \tau) d \tau \quad \text { (16) }
\end{aligned}
$$

where $j_{0}=\max (0, k-i)$ and

$$
\begin{aligned}
& A(\nu, n, \kappa, N)=\frac{(-1)^{n+\kappa}(\nu+n+\kappa) !}{(n+\kappa) !(N-n) !} \\
& B(i, n, N)=\left(\begin{array}{l}
N-n \\
i
\end{array}\right) \frac{(N+1) !}{(n+i+1) !} \\
& C(i, j, n, \kappa)=\left(\begin{array}{l}
n+\kappa \\
j
\end{array}\right) \frac{(n+i) !}{(i+j-\kappa) !}
\end{aligned}
$$

Remark 5: An $n^{\text {th }}$-order truncated Taylor expansion is appropriate for estimating the $n^{\text {th }}$-order derivative. ${ }^{6}$

Remark 6: The resulting derivative estimation is always subject to a time delay $\tau_{0}$ [22], defined as follows:

$$
\tau_{0}=\frac{\kappa+2}{\kappa+\nu+4} T
$$

which is needed to be compensated.

Remark 7: This algebraic differentiator can also remove noise, since that it only contains integrators which perform slightly as a low-pass filter. Corrupting noises are viewed as highly fluctuating phenomena, which are attenuated via low-pass filters (see [8] for more details). Such filters are realized by multiplying both sides of (15) by $s^{-v}$, where $v=N+1+\mu$ with $\mu>0$.

\section{ILLUSTRATIVE EXAMPLE}

This section is devoted to highlighting the feasibility of the proposed method. For this, let us consider directly a simple example which is already in the form (10) as follows:

$$
\left\{\begin{array}{l}
\dot{z}_{1}=z_{2}-z_{1} \\
\dot{z}_{2}=z_{3}-2 z_{1}+\beta(y) q \\
\dot{z}_{3}=z_{2}+z_{4} \\
\dot{z}_{4}=-z_{4}-z_{3} \\
y=z_{1}
\end{array}\right.
$$

with $\beta(y)=y$ and $q=\operatorname{sign}(w)$, where $w$ is a high frequency noise around 0 and this form of $q$ leads to a zeno phenomenon or at least hight frequency switching (see [1] for Zeno phenomena simulation). It is easy to check that Assumptions 1 and 2 are satisfied, hence algebraic observer can be applied for system (18) to estimate its states.

Based on the principal explained before, let us define $\chi$ according to (7) as follows:

$$
\chi(t)=\int_{t-T}^{t} \int_{s-T}^{s} y(\xi) q_{f}(\xi) d \xi d s
$$

and a new output can be defined

$$
Y=y-\chi
$$

${ }^{5}$ See [22] for details.

${ }^{6}$ See Lemma 1 in [22].
Thus, a straightforward calculation yields the estimations of $z_{1}$ and $z_{2}$ as follows:

$$
\left\{\begin{aligned}
z_{1} & =Y+\chi \\
z_{2} & =\dot{Y}+\dot{\chi}+z_{1} \\
& =\dot{Y}+\dot{\chi}+Y+\chi
\end{aligned}\right.
$$

and we have

$$
\begin{aligned}
z_{3} & =\ddot{Y}+\ddot{\chi}+z_{2}+z_{1}+(Y+\chi)\left(q_{f}-q\right) \\
& =\ddot{Y}+\ddot{\chi}+\dot{Y}+\dot{\chi}+2 Y+2 \chi+(Y+\chi)\left(q_{f}-q\right)
\end{aligned}
$$

By using algebraic observer, the estimate of $z_{3}$ can be obtained as

$$
z_{3 f}=\ddot{Y}+\ddot{\chi}+\dot{Y}+\dot{\chi}+2 Y+2 \chi
$$

Since the 3rd time derivative of $Y$ does not exist, hence we use the derivative of $z_{3 f}$, the estimate of $z_{3}$, to reconstruct $z_{4}$, which yields:

$$
\begin{aligned}
z_{4} & =\dot{z}_{3 f}-z_{2} \\
& =\dot{z}_{3 f}-\dot{Y}-\dot{\chi}-Y-\chi
\end{aligned}
$$

Based on the deduced equations, an algebraic observer can be applied to estimate $\dot{Y}, \ddot{Y}, \dot{\chi}, \ddot{\chi}$ and $\dot{z}_{3 f}$. It is necessary to fix a appropriate window size $T$ for the integrator and the algebraic differential operator, while we set this parameter as $T=0.8 \mathrm{~s}$ corresponding to the fixed time step $t_{\delta}=0.001 \mathrm{~s}$. For the other parameters in algebraic differential operator (16), $\nu=\kappa=2$, and $N=1$ for the first order derivative ( $N=2$ for the second order derivative). Moreover, since the zeno phenomenon cannot be simulated, hence in this example we approximate it by $q=\operatorname{sign}(w)$ where $w$ is a high frequency noise signal with the lowest frequency equal to $1 \mathrm{KHz}$. In the simulation, we use the fourth-order RungeKutta method to approximate the solution of ODEs with a fixed time step equal to $0.001 s$.

The simulation results are depicted in Fig. 1, where it is worthy mentioning that the incorrect estimation at the beginning is due to the time delay of numerical integrator and the numerical algebraic differential operator. For the inherent time delay (17) inside the algebraic differential operator, it can be calculated and has been compensated in the simulation results.

\section{CONClusion}

In this paper, we demonstrate a novel scheme to construct an observer for a large class of switched systems with zeno phenomenon. Since that the zeno terms are not differentiable, algebraic observer cannot be adopted directly. However, assuming that these terms are integrable, we can reconstruct the output and renew the discrete states to make these terms integrable in the final observer, where algebraic observer can be possibly utilized. Meanwhile, we have proved the equivalence between the novel observer with new output and states and the observer with original ones. An academical example is given to illustrate our theorems. 


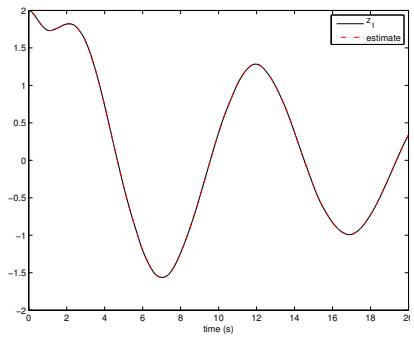

$A$

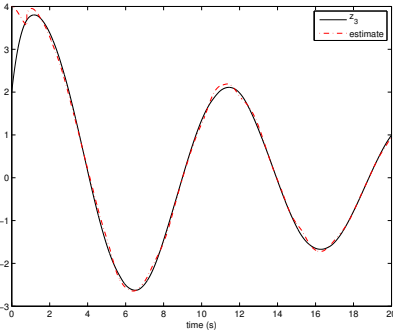

C

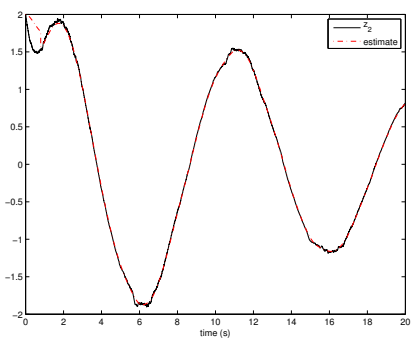

$B$

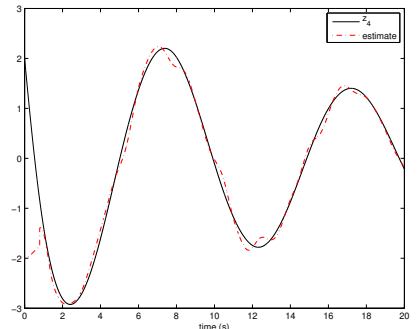

$D$
Fig. 1. A: $z_{1}$ and its estimate with $z_{1}(0)=2$; B: $z_{2}$ and its estimate with $z_{2}(0)=2$; C: $z_{3}$ and its estimate with $z_{3}(0)=4 ; \mathrm{D}: z_{4}$ and its estimate with $z_{4}(0)=-2$.

\section{REFERENCES}

[1] V. Acary and B. Brogliato, Numerical Methods for Nonsmooth Dynamical Systems: Applications in Mechanics and Electronics. Springer, 2008.

[2] A. Ames, H. Zheng, R. Gregg, and S. Sastry, "Is there life after zeno? taking executions past the breaking (zeno) point," in Proc. of IEEE American Control Conference, 2006.

[3] J.-P. Barbot, I. Belmouhoub, and L. Boutat-Baddas, "Observability normal form," LNCIS: New Trends in Nonlinear Dynamics and Control and their Applications, 2002.

[4] J.-P. Barbot, M. Fliess, and T. Floquet, "An algebraic framework for the design of nonlinear observers with unknown inputs," in Proc. of IEEE Conference on Decision and Control, pp. 384-389, 2007.

[5] M. Cichon, I. Kubiaczyk, and A. Sikorska, "The henstock-kurweilpettis integral and exsitence theorems for the cauchy problem," Czeckoslovak Mathematical Journal, vol. 54, pp. 279-289, 2004.

[6] S. Emelyanov and S. Korovin, "Applying the principle of control by deviation to extend the set of possible feedback types," Soviet Physics Doklady, vol. 26, no. 6, pp. 562-564.

[7] M. Fliess, C. Join, and H. Sira-Ramirez, "Non-linear estimation is easy," International Journal of Modelling Identification and Control, vol. 4, no. 1, pp. 12-27, 2008.

[8] M. Fliess and H. Sira-Ramirez, "Reconstructeurs d'état," Comptes Rendus de l'Académie des Sciences - Series I, vol. 338, no. 1, pp. 91-96, 2004

[9] M. Fliess, "Analyse non standard du bruit," Comptes Rendus de l'Académie des Sciences - Series I, vol. 342, no. 10, pp. 797-802, 2006.

[10] M. Fliess, C. Join, and W. Perruquetti, "Real-time estimation for switched linear systems," in Proc. of IEEE Conference on Decision and Control, 2008.

[11] M. Fliess, C. Join, and H. Sira-Ramirez, "Complex continuous nonlinear systems: their black box identification and their control," in Proc. of the 14th IFAC Symposium on System Identification, 2006.

[12] R. Goebel and R. Teel, "Lyapunov characterization of zeno behavior in hybrid systems," in Proc. of IEEE Conference on Decision and Control, 2008.

[13] H. Hammouri and J. Morales, "Observer synthesis for state-affine systems," in Proc. of IEEE Conference on Decision and Control, 1990.
[14] R. Henstock, The General Theory of Integration. Oxford Mathematical Monographs, Clarendon Press, 1991.

[15] M. Heymann, F. Lin, G. Meyer, and S. Resmerita, "Analysis of zeno behaviours in hybrid systems," in Proc. of IEEE Conference on Decision and Control, 2002.

[16] W. Kang, "Bifurcation and normal form of nonlinear control system: Part i and ii," SIAM Journal on Control and Optimization, vol. 36, pp. 193-232, 1998.

[17] A. Krener and A. Isidori, "Linearization by output injection and nonlinear observer," Systems \& Control Letters, vol. 3, pp. 47-52, 1983.

[18] A. Lamperski and A. Ames, "On the existence of zeno behavior in hybrid systems with non-isolated zeno equilibria," in Proc. of IEEE Conference on Decision and Control, 2008.

[19] A. Levant, "Sliding order and sliding accuracy in sliding mode control," International journal of control, vol. 58, no. 6, pp. 1247$1263,1993$.

[20] - "Homogeneity approach to high-order sliding mode design," Automatica, vol. 41, no. 5, pp. 823-830, 2005.

[21] D. Liberzon, Switching in system and control. Birkhauser, 2003.

[22] M. Mboup, C. Join, and M. Fliess, "A revised look at numerical differentiation with an application to nonlinear feedback control," in Proc. of the 15th Mediterranean Conference on Control and Automation, 2007.

[23] E. Pereira, J. Trapero, I. Díaz, and V. Feliu, "Adaptive input shaping for manoeuvring flexible structures using an algebraic identification technique," Automatica, doi:10.1026/j.automatica.2008.11.014, 2008.

[24] W. Perruquetti and J.-P. Barbot, Sliding mode control in engineering. Marcel Dekker, New York, 2002.

[25] H. Sira-Ramirez and M. Fliess, "An algebraic state estimation approach for the recovery of chaotically encrypted messages," International Journal of Bifurcation and Chaos, vol. 16, no. 2, pp. 295-309, 2006.

[26] E. Talvila, "Estimates of the remainder in taylor's theorem using the henstock," Czeckoslovak Mathematical Journal, vol. 55, pp. 933-940, 2005.

[27] V. Utkin, Sliding Modes in Optimization and Control. SpringerVerlag, 1992.

[28] X. Xia and W. Gao, "Nonlinear observer design by observer error linearization," SIAM Journal on Control and Optimization, vol. 27, pp. 199-216, 1989

[29] L. Yu, J.-P. Barbot, D. Boutat, and D. Benmerzouk, "Observability normal forms for a class of switched systems with zeno phenomena," in Proc. of IEEE American Control Conference, 2009.

[30] G. Zheng, W. Aggoune, and J.-P. Barbot, "Praivate communication based on hybrid chaotic system with delays," in Proc. of the 2nd IFAC conference on Chaos, 2009.

[31] G. Zheng, D. Boutat, and J.-P. Barbot, "Single output dependent observability normal form," SIAM Journal on Control and Optimization, vol. 46, no. 6, pp. 2242-2255, 2007. 